\title{
Experimental investigation of physical mechanisms underlying lateral current injection laser operation
}

\author{
Edward H. Sargent ${ }^{\mathrm{a})}$ and J. M. Xu \\ Department of Electrical and Computer Engineering, University of Toronto, Toronto, M5S 1A4, Canada
}

Catherine Caneau and ChungEn Zah

Corning Inc., Red Bank, New Jersey 07701

(Received 26 February 1998; accepted for publication 14 May 1998)

\begin{abstract}
By comparing theoretical prediction with experimental performance, we gain insight into the physical operation of lateral current injection lasers. By studying the temperature dependence of lasing stimulated efficiency, we demonstrate the influence of lateral heterobarriers (achieved in this experiment by quantum well intermixing) on the confinement of carriers to the active region. By comparing the evolution of threshold current with temperature for devices of differing contact separations with a self-consistent model of lateral current injection laser operation, we reveal the importance of the interplay between the lateral material gain profile and the optical mode. Understanding these mechanisms, unique to the lateral injection family of lasers, is key to realizing the tremendous potential of this class of lasers to enable optoelectronic integration and novel functional devices. (C) 1998 American Institute of Physics. [S0003-6951(98)02529-7]
\end{abstract}

An active source for monolithic optoelectronic integration should consist ideally of predominantly undoped epitaxial layers for ease of interdevice isolation and low freecarrier absorption in passive sections, be grown on semiinsulating material for low parasitic conduction and delay, have both contacts on the top side of the substrate for ease of interconnection, and be readily fabricated using a planar process. Vertical injection lasers, though their performance as discrete devices is greatly perfected, do not generally possess these characteristics. Lateral current injection (LCI) lasers, while they have previously been recognized as natural candidates for integration and novel functionality, remain to be studied systematically and developed to their full potential. Experimental LCI laser trials have often exhibited disappointing performance, ${ }^{1-7}$ though some attempts have been promising, showing performance comparable with conventional vertical injection lasers ${ }^{8}$ together with the benefits of lateral injection.

We have initiated theoretical studies aimed at understanding the physical mechanisms which underlie LCI laser operation. ${ }^{9-14}$ We have shown laterally nonuniform carrier density profiles in LCI lasers, which become severe when the active region width exceeds the ambipolar diffusion length of carriers, to give rise to reduced overlap between the fundamental lateral optical mode and the material gain profile. ${ }^{11,12}$ We have demonstrated lateral heterobarrier formation to be necessary for low-threshold, high-efficiency LCI laser operation, finding that in a well-designed LCI laser, recombination current will dominate over leakage only if the barrier is of a height of at least $4 \mathrm{kT}{ }^{9}$ We have shown parallel leakage to become severe at medium and high injection levels if measures are not taken to reduce active region series resistance, even if prospective leakage paths are un-

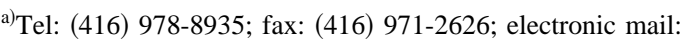
sargente@vrg.toronto.edu
}

doped and of a substantially higher band gap. ${ }^{14}$ Finally, the vulnerability of LCI lasers to Joule heating is evident by comparison of cw and pulsed performance (e.g., Ref. 15).

We fabricated a set of LCI lasers in order to study these mechanisms and enable comparison of theory and experiment. We employed ion implantation, an attractive technique for spatially selective doping and/or intermixing of semiconductor heterostructures. By promoting the alloying of adjacent heteroepitaxial layers (e.g., quantum wells and barriers), vacancies introduced during the course of ion implantation enable regrowth-free formation of the lateral heterobarriers required in LCI lasers for adequate current confinement. ${ }^{9}$

The epitaxial structure was grown by organometallic vapor phase epitaxy on InP:Fe. The bottom cladding consisted of $1 \mu \mathrm{m} \mathrm{InP:Fe}$. The active region, with photoluminescence peak at $1.55 \mu \mathrm{m}$, was made up of ten $95 \mathrm{~A} 1 \%$ compressively strained undoped wells separated by 190 A $0.5 \%$ tensile strained $\mathrm{Ga}_{0.18} \mathrm{In}_{0.82} \mathrm{As}_{0.25} \mathrm{P}_{0.75}(Q=1.1 \mu \mathrm{m}) \quad p$-type $5 \times 10^{17} \mathrm{~cm}^{-3}$ barriers. Above and below this multiquantum well (MQW) structure were 500 A thick layers of unstrained GaInAsP $(Q=1.07 \mu \mathrm{m}) \quad$ doped $p$-type $5 \times 10^{17}$ and $1 \times 10^{18} \mathrm{~cm}^{-3}$, respectively. The top cladding consisted of $0.5 \mu \mathrm{m}$ undoped InP, on top of which was grown a $0.2 \mu \mathrm{m}$ thick contact layer of undoped $\mathrm{Ga}_{0.47} \mathrm{In}_{0.53}$ As. Iron doping was used in the substrate and lower cladding to reduce parallel leakage current. Compressively strained quantum wells were used to minimize Auger recombination and break the heavy hole-light hole degeneracy, thereby reducing the heavy hole effective mass and increasing the hole mobility (and therefore the ambipolar diffusion length). Current guiding layers above and below the MQW, along with the thick $p$-doped barriers, served to reduce the series resistance of the active region by promoting hybrid injection of holes.

The processing sequence carried out is depicted in Fig. 1. The GaInAs contact layer was removed using a selective wet etch in the vicinity of the future ridge [Fig. 1(a)] in order to avoid optical absorption of the lasing mode. $\mathrm{SiO}_{2}$ was 


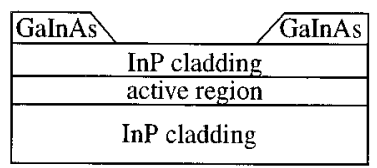

(a) etch contact layer

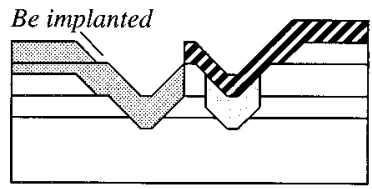

(c) implant p-type dopant

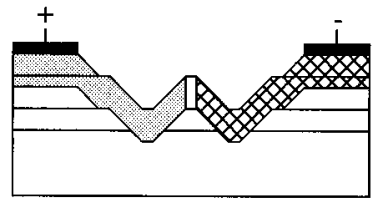

(e) metallize

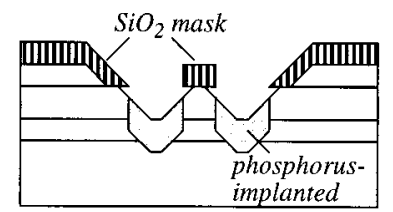

(b) etch ridges; implant phosphorus

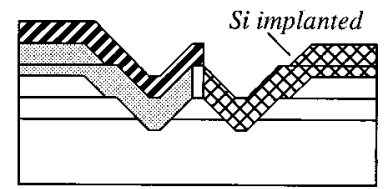

(d) implant n-type dopant

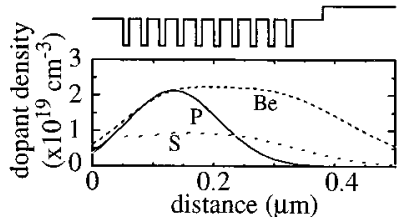

(f) calculated implant profiles.
FIG. 1. Processing sequence.

deposited and patterned; ridges were formed using a selective wet etch, stopping on the upper GaInAsP current guide [Fig. 1(b)]. The $\mathrm{SiO}_{2}$ served as a self-aligned mask for selective implantation of phosphorus into the quantum wells with a dose of $2 \times 10^{14} \mathrm{~cm}^{-2}$. The dielectric mask was then stripped and the samples subjected to rapid thermal annealing in the proximity geometry in order to intermix the wells [Fig. 1(c)]. Samples were masked to reveal the intended $n$ contact regions [Fig. 1(d)] and implanted with silicon, then masked for implantation of beryllium in order to form the $p$-contacts [Fig. 1(c)]. Another anneal was performed, also using an InP proximity cap, in order to activate the implanted dopant ions. Ti/Pt/Au was evaporated for the $p$-contact while $\mathrm{Ni} / \mathrm{Au} / \mathrm{Ge} / \mathrm{Au}$ was evaporated for the $n$-contact. Samples were cleaved into $500 \mu \mathrm{m}$ bars and mounted on aluminum nitride carriers.

The devices lased only at low temperatures. While the ultimate objective of LCI laser fabrication is to realize room temperature, continuous wave, low threshold, high efficiency lasing, this experimental trial permitted us nevertheless to study the physical mechanisms which underlie LCI laser operation. Our temperature-dependent probing of the performance of the LCI lasers fabricated permitted us to reveal which mechanisms prevented successful room temperature lasing of these devices and to propose technologically feasible design and processing improvements.

We first studied the dependence of measured output parameters on pulse width and duty cycle and identified a region over which device heating was not significant. We show in Fig. 2 the measured evolution of the stimulated emission efficiency of this laser as a function of heatsink temperature. We fit this to a model of stimulated efficiency which accounts for the temperature dependence of the various recombination mechanisms and of leakage over the heterobarriers. Using typical values for material parameters (mobility with $T^{-3 / 2}$ dependence and room temperature value $\mu_{e}$ $=3500 \mathrm{~cm}^{2} / \mathrm{V} \mathrm{s} ;{ }^{16}$ quantum well bimolecular coefficient with $T^{-1}$ dependence and room temperature value $B=0.9 \times 10^{-10} \mathrm{~cm}^{3} \mathrm{~s}^{-1}$ (Ref. 17); and threshold carrier density temperature dependence which varies as $T$ ), we estimate

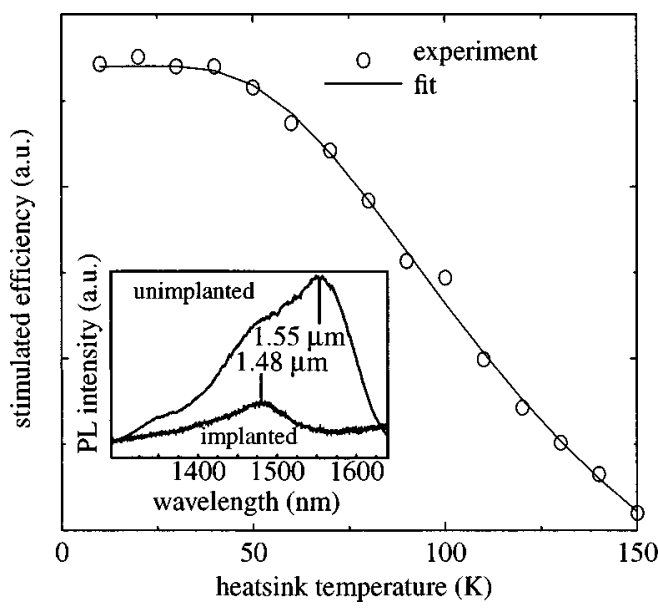

FIG. 2. Stimulated efficiency vs heatsink temperature for a device with a 3 $\mu \mathrm{m}$ wide ridge.

the degree of lateral heterobarrier formation obtained by implanting and annealing to have been around $30 \mathrm{meV}$. This is in reasonable agreement with the measured roomtemperature photoluminescence spectral shift of $38 \mathrm{meV}$ for implanted versus unimplanted materials annealed under the same conditions (inset of Fig. 2). Thus, one main reason for nonlasing at room temperature was the insufficient degree of quantum well intermixing: for room temperature operation, our $4 \mathrm{kT}$ rule of thumb would imply that more than $100 \mathrm{meV}$ of effective bandgap shift in the quantum well region is required. Significantly greater intermixing (preferably up to $200 \mathrm{meV}$ ) would enable improved efficiency and better highpower operation. Ion implantation and annealing in the InGaAs/InP materials system have previously been shown experimentally to allow band-gap shifts as large as 200 meV. ${ }^{18}$ The intermixing of thinner wells and thick, highband-gap barriers would enable the needed amount of carrier confinement.

We compare in Fig. 3 the temperature dependence of threshold current for lasers with 3 and $1 \mu \mathrm{m}$ wide ridges. The fact that the threshold current is appreciably lower for the wider-ridge devices would suggest that the $1 \mu \mathrm{m}$ wide device experiences a much larger scattering loss. There is a discontinuous change in the slope of the threshold current temperature dependence for the $3 \mu \mathrm{m}$ wide device around 50

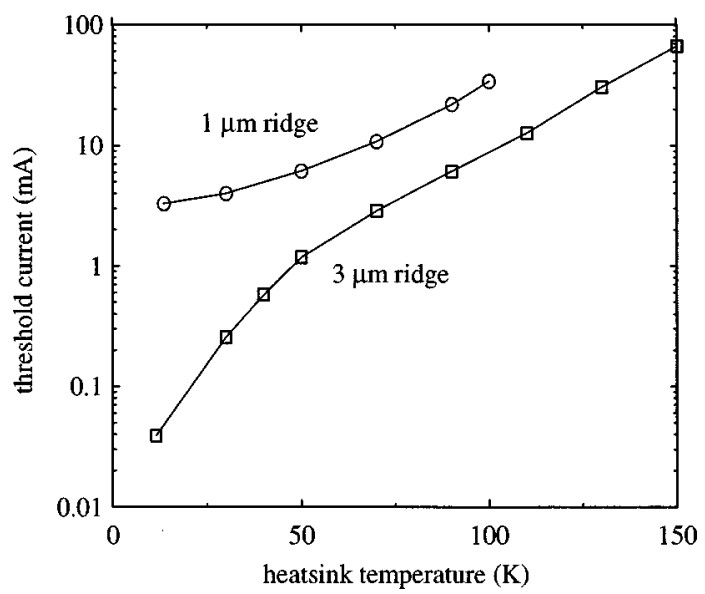

FIG. 3. Measured threshold current vs heatsink temperature for devices with ridge widths of 1 and $3 \mu \mathrm{m}$. 


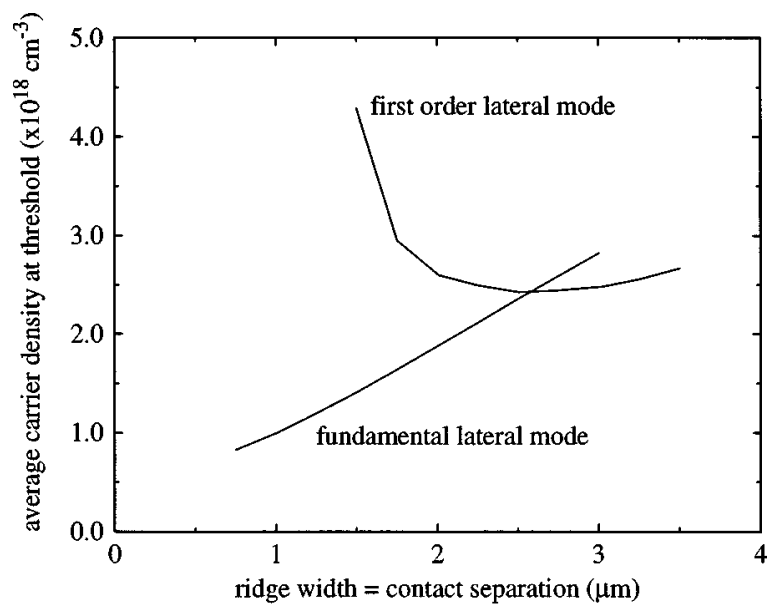

FIG. 4. Calculated average carrier density at threshold vs ridge width (which is equal in this case to the separation of the $p$ - and $n$-contacts). It is thus predicted that, for ridge widths in excess of $2.6 \mu \mathrm{m}$, the first-order mode will exhibit greater overlap with the laterally nonuniform material gain profile and will therefore lase first.

$\mathrm{K}$; this coincides with a change in the far field from firstorder transverse mode lasing below $50 \mathrm{~K}$ to fundamental mode lasing above this temperature. This observation, and the lack of an analogous discontinuity in the case of the 1 $\mu \mathrm{m}$ wide laser, can be explained with reference to the laterally nonuniform carrier density distribution. If the ambipolar diffusion length, $L_{a}$, is constant, the carrier density distribution may be written, ${ }^{19}$

$$
n(x)=\bar{n}\left[\frac{\cosh \left(x / L_{a}\right)}{\sinh \left(d / L_{a}\right)}-\frac{b-1}{b+1} \frac{\sinh \left(x / L_{a}\right)}{\cosh \left(d / L_{a}\right)}\right],
$$

where $b$ is the mobility ratio $\mu_{e} / \mu_{h}, \bar{n}$ is the average carrier density, and the active region extends from $x=-d$ to $x=d$. We used the effective index method to determine the lateral wave vectors and confinement factors and calculated the gain seen by each mode. We show in Fig. 4 the calculated average threshold carrier density $\bar{n}_{\text {th }}$ (proportional to the threshold current in this simple approximation) as a function of ridge width for the fundamental and first-order modes, assuming $L_{a} \sim 1 \mu \mathrm{m}$ and $\mu_{e} / \mu_{h} \sim 20$ ). If the lateral contact separation is large enough for the lateral carrier density profile to be strongly nonuniform, and if the lateral confinement factors for the fundamental and first-order modes are similar, then the first-order mode may lase first (assuming modal losses are approximately equal) because of its greater overlap with the lateral modal gain profile. The quantitative details of the ridge width at which this transition occurs will of course depend (at any given temperature) on the ambipolar diffusion length, electron-hole mobility ratio, and lateral modal profiles.

On the basis of this finding, we argue that decreased low-temperature mobility arising as a result of increased ionized impurity scattering below $\sim 50 \mathrm{~K}$ gives rise to a reduced ambipolar diffusion length. This decrease in the ambipolar diffusion length enables first-order mode lasing in the $3 \mu \mathrm{m}$ wide ridge device prior to fundamental mode lasing. In the 1 $\mu \mathrm{m}$ wide ridge device, there is no first-order lateral mode admitted (or its confinement factor is very small), so lasing always occurs first on the fundamental mode. However, as the ambipolar diffusion length drops at low temperatures, the threshold current drops more slowly as gain-mode overlap worsens with increasing lateral carrier density nonuniformity.

By comparing measured device behavior with the results of physical modeling, we have revealed two key features of lateral current injection lasers. We demonstrated the relationship between stimulated efficiency and lateral heterobarrier formation, finding that a large but realizable band-gap shift achievable by ion mixing should enable room temperature LCI laser operation. We reported an experimental manifestation of the relationship between ambipolar diffusion length and lateral optical mode profiles, another key feature in LCI lasers. Continual development of the understanding of the issues highlighted in this letter is essential to achieving further insight into the physical mechanisms underlying LCI laser operation and, in turn, to realizing the tremendous potential of LCI lasers to enable OEICs and novel functional devices.

${ }^{1}$ Y. Kan, M. Yamanishi, M. Okuda, K. Mukaiyama, T. Ohnishi, M. Kawamoto, and I. Suemune, Appl. Phys. Lett. 55, 1149 (1989).

${ }^{2}$ I. Suemune, T. Takeoka, M. Yamanishi, and Y. Lee, IEEE J. Quantum Electron. QE-22, 1900 (1986).

${ }^{3}$ A. Furuya, M. Makiuchi, O. Wada, and T. Fujii, IEEE J. Quantum Electron. 24, 2448 (1988).

${ }^{4}$ Y. Honda, I. Suemune, N. Yahuira, and M. Yamanishi, Jpn. J. Appl. Phys., Part 1 30, 990 (1991).

${ }^{5}$ J. Ohta, K. Kuroda, K. Mitsunaga, K. Kyuma, K. Hamanaka, and T. Nakayama, J. Appl. Phys. 61, 4933 (1987).

${ }^{6}$ Y. Kawamura, Y. Noguchi, and H. Iwamura, Electron. Lett. 29, 102 (1993).

${ }^{7}$ Y. Suzuki, S. Mukai, H. Yajima, and T. Sato, Electron. Lett. 23, 384 (1987).

${ }^{8}$ K. Oe, Y. Noguchi, and C. Caneau, IEEE Photonics Technol. Lett. 6, 479 (1994).

${ }^{9}$ E. H. Sargent and J. M. Xu, Proc. CLEO/Pacfic Rim, 1997.

${ }^{10}$ E. H. Sargent, G. Tan, and J. M. Xu, IEEE J. Sel. Top. Quantum Electron. 3, 507 (1997).

${ }^{11}$ E. H. Sargent and J. M. Xu, Proc. IEEE LEOS Annual Meeting, 1996.

${ }^{12}$ E. H. Sargent, G. L. Tan, D. A. Suda, and J. M. Xu, Proc. IEEE ISLC, 1996.

${ }^{13}$ E. H. Sargent, G. Tan, and J. M. Xu, Proc. ISCS, 1996.

${ }^{14}$ E. H. Sargent, K. Oe, C. Caneau, and J. M. Xu, IEEE J. Quantum Electron. (to be published).

${ }^{15}$ W. Yang, A. Gopinath, and M. Hibbs-Brenner, IEEE J. Sel. Top. Quantum Electron. 7, 848 (1995).

${ }^{16}$ Properties of Lattice-matched and Strained Indium Gallium Arsenide, edited by P. K. Bhattacharya (INSPEC, London, 1993).

${ }^{17}$ R. F. Kazarinov and M. R. Pinto, IEEE J. Quantum Electron. 30, 49 (1994).

${ }^{18}$ J. Z. Wan, J. G. Simmons, and D. A. Thompson, J. Appl. Phys. 81, 765 (1997).

${ }^{19}$ A. Herlet, Solid-State Electron. 11, 717 (1968). 